\title{
The natural course of cow's milk protein allergy and atopic diseases in a birth cohort with follow-up into adulthood
}

\author{
Michaela Manalili Hansen ${ }^{1}$, Arne Høst ${ }^{2}$, Susanne Halken², and Susanne Nissen² \\ ${ }^{1}$ University of Southern Denmark \\ ${ }^{2}$ Odense University Hospital
}

June 22, 2020

\begin{abstract}
Background: Previous studies have investigated the natural course of cow's milk protein allergy (CMPA) and development of atopic diseases into adolescence. Studies with long term follow-up into adulthood are lacking. The aim of this study was to investigate 1) the natural course of CMPA in a 1-year birth cohort of Danish children from birth until 15 and 26 years of age and 2) the development of atopic diseases in a group of children with CMPA (group A) compared to a random sample of 276 children from the same birth cohort (group B). Methods: A birth cohort of 1,749 newborns, was investigated prospectively for the development of CMPA and atopic diseases. During the first year of life and at 18 months and 3, 5, 10, 15, and 26 years of age, questionnaire based interviews, physical examination, skin prick tests and specific $\operatorname{IgE}$ testing, and from 10 years also spirometry, were carried out. Results: 39 (2,2\%) were diagnosed with CMPA. The recovery rate was $87 \%, 92 \%$ and $97 \%$ at 3, 5 and 26 years of age. Compared to group B, group A had significantly $(\mathrm{p}<0,05)$ higher prevalence of asthma and rhinoconjunctivitis at 15 years of age and at 26 years of age, group A had significantly higher prevalence of asthma and atopic dermatitis. The follow-up rate was $85 \%$ (A) and $70 \%$ (B). Conclusion: CMPA has a good prognosis regarding recovery rate. CMPA and sensitization in early childhood predict sensitization and persistence of allergic diseases into adulthood.
\end{abstract}

\section{Introduction}

Cow's milk protein allergy (CMPA) is the most common food allergy in infants, with incidence rates estimated between $2 \%$ and $3 \%$ in developed countries (1-4). The majority of infants with CMPA develop symptoms before one month of age. CMPA debuting after 12 months of age is extremely rare $(2,3)$. Allergic reactions to cow's milk proteins (CMP) are most often IgE-mediated, causing cutaneous, gastrointestinal and respiratory symptoms. Food allergy manifestations may vary from clearly IgE-mediated to mixed reactions dominated by eosinophilic granulocytes as effector cells or clearly non-IgE mediated reactions. A differentiation between IgE-mediated and non-IgE mediated reactions to CMP cannot be made based on clinical symptoms alone (3). Studies have shown that infants with IgE sentitization to CMP have an increased risk of persisting CMPA, and are more likely to develop persisting allergy to other foods and inhalant allergies before three years of age $(1,3,4)$. Food allergy (FA) and atopic dermatitis (AD) dominate in early childhood, while allergic asthma (AA) and rhinoconjunctivitis (RC) are more common later. This progression from FA and $\mathrm{AD}$ to $\mathrm{AA}$ and subsequently to $\mathrm{RC}$ is known as the atopic march (5).

Longitudinal prospective birth cohort studies provide a desciption of the natural course of allergic diseases and sensitization. There are very few longitudinal studies on the natural history of CMPA and the long term development of atopic diseases in children diagnosed with CMPA. The studies conducted on the natural course of CMPA report varying recovery rates, most likely due to the differences in methods and design.

From the 1-year birth cohort in this study, the clinical course of CMPA regarding remission rate during the 
first 15 years of life has previously been reported in detail $(1,6)$. Clinical, epidemiological and immunological aspects of CMPA in the same study population have been reviewed and evaluated in relation to other studies on this subject (3). A 26 year follow-up on the natural course of sensitization and allergic diseases in a random sample of 276 children (16\%) from the same birth cohort has previously been reported (7). Further-more, other studies from the same birth cohort have been published (8-12).

The aim of this study was to investigate 1) the natural course of CMPA in a 1-year birth cohort of Danish children from birth until 15 and 26 years of age and 2) compare the development of atopic diseases in a group of children with CMPA (group A) with the development of atopic diseases in a random sample of 276 children from the same birth cohort (group B).

\section{Methods}

A cohort of 1,749 newborns, born during 1985 at the Odense University Hospital, was followed up prospectively for the development of CMPA during their first year of life. The infants were referred to the paediatric clinic at the hospital when one or more of the following symptoms occurred: Recurrent wheezing, rhinitis, atopic eczema, urticaria, erythema/exanthema, vomiting and/or diarrhoea not caused by coincidental infections or other demonstrable causes; failure to thrive and infantile colic not disappearing after advice on feeding technique. This part of the study has previously been described in detail $(1,8)$.

The diagnosis of CMPA was established by the following, generally accepted, criteria $(1,3,6,13,14)$ : a definite disappearance of symptoms after each of two dietary eliminations of cow's milk and cow's milk products; recurrence of identical symptoms after one challenge; and exclusion of lactose intolerance and coincidental infection.

Details on elimination and challenge procedures have previously been described in full $(1,3,15)$. Once a diagnosis of CMPA was confirmed, the milk-free diet was continued until a new milk challenge had shown development of tolerance to cow's milk protein. All infants with confirmed CMPA were rechallenged at 12 months of age and the infants with non-confirmed CMPA (negative milk challenge) were also reviewed at 12 months of age. In the event of continued clinical sensitivity to cow's milk protein, rechallenges were performed at 18, 24, and 36 months of age. Infants with CMPA during the first year of life were investigated every 6 months until 3 years of age. Thereafter, re-challenge was performed every 12 months until 15 years of age, to assess continued clinical sensitivity. The one remaining subject with CMPA from 15 to 26 years of age, was not rechallenged further, due to the severity of symptoms persisting until 26 years of age.

SPT (ALK Soluprick®), standard panel) (16) and specific sIgE testing (CAP RAST at 18 months, 3, 5, and 10 years and UniCAP at 15 and 26 years; Pharmacia Diagnostics, Uppsala, Sweden) (17) were carried out against inhalant allergens and food allergens in all children with CMPA at 18 months, 3, 5, 10, 15, and 26 years of age. Furthermore, lung function was measured by spirometry at 10, 15 and 26 years of age.

Based on the results of SPT and specific SIgE testing to cow's milk, the reactions were immuno-logically classified as A) IgE-mediated CMPA when SPT was [?] 2+ and/or RAST [?] class 2 or B) non-IgE-mediated CMPA when SPT $<2+$ and RAST $<$ class 2. Method for classification of SPT and RAST has been described in detail by Host et. al. (1).

From the same birth cohort, a population based sample of 276 (16\%) was randomly selected for prospective follow-up of atopic diseases $(6,7)$.

At 0,6 and 12 months the parents filled in a questionnaire regarding atopic heredity, environ-mental factors, respiratory tract infections, wheezing, eczema, adverse reaction to foods and other possible signs of allergic disease. At ages 18 months, 5, 10, 15, and 26 years, all participants underwent physical examination by a doctor at Hans Christian Andersen Children's Hospital, Odense University Hospital, and were interviewed regarding the same factors as in the before-mentioned questionnaire.

Specific sIgE testing was also carried out in children at 18 months, 5, 10, 15, and 26 years of age (CAP RAST at 18 months, 5 and 10 years and UniCAP at 15 and 26 years; Pharmacia Diagnostics, Uppsala, Sweden). 
Lung function was measured by spirometry at 10, 15, and 26 years of age. $(6,7)$

The study population of children with CMPA (group A) was compared to the population based sample (group B) regarding the current prevalence of atopic diseases; AD, AA and RC.

\section{Definitions and diagnostic criteria}

\section{Atopic dermatitis}

Areas of scaly, erythematous and itchy eczematous rash primarily of the face and scalp, behind the ears and at the flexural folds, diagnosed by a doctor. Only eczema localized to at least two typical areas and chronically relapsing with duration of at least 3 months were recorded.

\section{Asthma}

Diagnosed by a physician by at least three episodes and more than two of the symptoms: wheeze, cough or dyspnea, not only associated with respiratory infections, responding to bronchodilator and/or inhaled corticosteroid treatment. From school age, the diagnosis was confirmed by variation in lung function spontaneously or due to therapy, for example an increase in FEV1 [?]12\% after inhalation of a beta-2-agonist and/or long term profylactic inhaled steroid.

\section{Rhinoconjunctivitis}

Physician diagnosed typical symptoms lasting at least 1 month or recurring on exposure to the same allergen. Sensitization

Defined as specific IgE [?]0.35 kU/l (CAP RAST/UniCAP; Pharmacia Diagnostics).

\section{Ethics}

The study was conducted according to the latest version of the Declaration of Helsinki for biomedical research involving humans and approved by the Regional Scientific Ethical Committee for Southern Denmark.

\section{Statistics}

Data management and statistical analyses were performed using IBM SPSS Statistics version 26 for macOS. For comparison of groups the chi-square test $\left(\chi^{2}\right)$ was used. The p-value of significance was $<0.05$.

\section{Results}

The follow-up rate of group A was $85 \%$ at 15 and 26 years. The follow-up rate of group B was $78 \%$ at 15 years and $70 \%$ at 26 years. Nissen et al. has tested the differences in distribution, to examine whether the group with incomplete follow-up differed from the group with complete follow-up of the subjects in group B (7). The only significant difference $(\mathrm{p}<0.05)$ was found in a higher prevalence of eczema $(17 \%)$ amongst the infants with full follow-up compared to the group with incomplete follow-up (7\%) (7).

During the first year of life, 117 infants $(6.7 \%)$ of the birth cohort of 1,749 infants had symptoms suggestive of CMPA. Based on strict elimination/milk-challenge procedures in a hospital setting, the diagnosis of CMPA was confirmed in 39 infants, giving a 1-year incidence of $2.2 \%$ (95\% CI: 1.5-2.9). Of the 39 infants with CMPA, 21 had IgE-mediated CMPA (positive skin-prick test and/or radioallergosorbent test (RAST) of ?class 2 to cow's milk protein) and 18 non-IgE-mediated CMPA. Symptoms of CMPA consisted of cutaneous symptoms (64\%), gastrointestinal symptoms (59\%) and respiratory symptoms (33\%). 36/39 (92\%) had more than one symptom $(3,6)$.

Table 1 presents the total recovery rates of CMPA from 1 to 26 years of age. There was full follow-up of the subject with persisting CMPA at 15 and 26 years.

At 15 years of age the prevalence of $\mathrm{AA}$ and $\mathrm{RC}$ was significantly higher $(\mathrm{p}=0.017$ and 0.002 , respectively) amongst children with IgE-mediated CMPA compared to children with non-IgE-mediated CMPA. At 26 years of age, children with IgE-mediated CMPA had a significantly higher prevalence of AA $(p=0.002)$ compared 
to children with non-IgE-mediated CMPA. There was not observed any significant difference between children with IgE-mediated CMPA and children with non-IgE-mediated CMPA at either age regarding AD.

The prevalence of atopic diseases at 15 and 26 years amongst children diagnosed with CMPA during their first year of life (group A) and children from the unselected group of infants (group B) are presented in table 2 .

The development of AA, RC and adverse reactions to other foods until 10 years of age has previously been reported in full $(1,6)$.

\section{Discussion}

This study describes the recovery rate of CMPA until 26 years of age and the prevalence of atopic diseases at 15 and 26 years of age in a birth cohort from 1985. Furthermore, it highlights the significant $(\mathrm{p}<0.05)$ differences in development of atopic diseases in a group of children with CMPA compared to a sample of unselected children from the same birth cohort.

\section{Recovery rate}

This longitudinal study with follow-up until 26 years of age of 39 infants with confirmed CMPA during their first year of life has shown that the overall prognosis of CMPA was good, with a total recovery of $87 \%$ at 3 years, $92 \%$ at 5 years and $97 \%$ at 15 and 26 years of age. The recovery rate for IgE-mediated CMPA alone was $76 \%$ at 3 years, $86 \%$ at 5 years and $95 \%$ at 15 and 26 years of age. In previously repported studies, the recovery rates for IgE-mediated CMPA have varied immensely (18-24).

The varying differences in recovery rates amongst children with CMPA are most likely related to the studied population and study design. Population based studies $(1,6,18,19)$ report a higher recovery rate of CMPA compared to studies based on selected patient materials from tertiary care referral centers (20-24).

\section{Prevalence of atopic diseases}

Many studies have been reported on the natural course of CMPA, risk factors for CMPA, recovery rates and on predictive factors of persisting CMPA. To our knowledge, there are no prior longitudinal studies spanning decades on the natural history of atopic diseases in children diagnosed with CMPA during the first year of life. Compared to the group of randomly selected children, children with CMPA had significantly $(\mathrm{p}<0.05)$ higher prevalence of asthma and rhinoconjunctivitis at 15 years of age. At 26 years of age, children with CMPA had significantly $(\mathrm{p}<0.05)$ higher current prevalence of astma and atopic dermatitis.

The concept of an atopic march, which explains the apparent progression of allergic diseases from AD to AA and to RC, is supported by the findings in both groups.

The atopic march hypothesizes that manifestation of allergic diseases begins in early childhood with the development of $\mathrm{AD}$, then $\mathrm{AA}$, progressing finally to $\mathrm{RC}(5)$. The 26 year follow-up in this study suggests, that allergic diseases such as $\mathrm{AD}$ and AA persist until adulthood in children diagnosed with CMPA during their first year of life.

\section{Limitations and strenghts}

It is difficult to avoid low follow-up rates in birth cohorts with long term follow-up. Yet in our study, the follow-up rate was considerably high at $85 \%$ for group A and $78 \%$ and $70 \%$ for group B, at 15 and 26 years, respectively. A limitation in our study was a small sample size. However, we have well defined sample of both infants with CMPA and a random sample of children from the same 1-year brth cohort. And all participants were followed closely and carefully in childhood up to 15 years of age and finally into adulthood at 26 years of age.

Participation in a cohort study may cause the participants to avoid possible risk factors due to increased awareness of allergic diseases, resulting in an underestimation of prevalence. Concurrently, an overestimation 
of prevalence amongst those with complete follow-up should be considered, since subjects with allergic diseases may be most motivated to participate in the study

We consider our data to have good validity and the results to be generalizable to the Danish population. All clinical investigations were performed or supervised by the same two pediatric allergologists ( $\mathrm{SH}$ and $\mathrm{AH}$ ) and included a rigorous methodology. We used predefined, generally accepted diagnostic criteria.

\section{Conclusion}

CMPA has a good prognosis regarding recovery rate. CMPA and sensitization in early childhood predict sensitization as well as development and often persistence of allergic diseases later in childhood and into adulthood. Children with CMPA exhibit a higher prevalence of asthma and rhinoconjunctivitis at 15 years of age, and at 26 years of age a higher prevalence of asthma and atopic dermatitis.

\section{Conflict of interest}

The authors declare no conflict of interest in relation to this article.

\section{Acknowledgements}

We thank the children, now adults, and their parents who participated in the Odense 1985 birth cohort study, and all staff involved in the study over the years. The study was supported by grants from A. J. Andersen og Hustrus Fond, Kong Christian Den Tiendes Fond, Civilingenior Frode Nyegaard og hustrus Fond, Aase og Ejnar Danielsens Fond, Fonden til Laegevidenskabens Fremme, Odense University Hospital, Phadia, Statens Laege-videnskabelige Forskningsrad, and Chresten Otto Hansen og hustrus fond.

v. Acknowledgements We thank the children, now adults, and their parents who participated in the Odense 1985 birth cohort study, and all staff involved in the study over the years.

\section{vi. Impact statement}

This is the first longitudinal birth cohort study on children diagnosed with cow's milk protein allergy (CMPA) with follow-up in adulthood at 26 years of age. This study reports the recovery rate of CMPA until 26 years of age, as well as the prevalence of atopic diseases at 15 and 26 years of age in children diagnosed with CMPA during the first year of life. Furthermore, the prevalence of atopic diseases amongst children diagnosed with CMPA during the first year of life is compared to the prevalence of the same diseases amongst a random sample of children from the same birth cohort. The children diagnosed with CMPA show a higher prevalence of astma/rhinoconjunctivitis and astma/atopic dermatitis at 15 and 26 years of age, respectively. This indicates that children who are sensitised from an early age, have a higher risk of developing and/or having persisting atopic diseases all the way into adulthood, and not just into childhood and adolescence as reported in previous studies. Data from follow up of the same birth cohort at 3 and 10 years has previously been published in Pediatric Allergy and Immunology.

vii. References

Host A, Halken S. A prospective study of cow milk allergy in Danish infants during the first 3 years of life. Clinical course in relation to clinical and immunological type of hypersensitivity reaction. Allergy. 1990 Nov;45(8):587-96.

Sicherer SH. Epidemiology of food allergy. J Allergy Clin Immunol. 2011 Mar;127(3):594-602.

Host A. Cow's milk protein allergy and intolerance in infancy. Some clinical, epidemiological and immunological aspects. Pediatr Allergy Immunol. 1994;5(5 Suppl):1-36.

Host A. Frequency of cow's milk allergy in childhood. Ann Allergy Asthma Immunol. 2002 Dec;89(6 Suppl 1):33-7.

Aw M, Penn J, Gauvreau GM, Lima H, Sehmi R. Atopic March: Collegium Internationale Allergologicum Update 2020. Int Arch Allergy Immunol. 2020;181(1):1-10. 
Host A, Halken S, Jacobsen HP, Christensen AE, Herskind AM, Plesner K. Clinical course of cow's milk protein allergy/intolerance and atopic diseases in childhood. Pediatr Allergy Immunol. 2002;13(s15):23-8.

Nissen SP, Kjaer HF, Host A, Nielsen J, Halken S. The natural course of sensitization and allergic diseases from childhood to adulthood. Pediatr Allergy Immunol. 2013 Sep;24(6):549-55.

Host A, Husby S, Osterballe O. A prospective study of cow's milk allergy in exclusively breast-fed infants. Acta Paediatr Scand 1988: 77: 663-70.

Halken S, Host A, Husby S, Hansen LG, Osterballe O, Nyboe J. Recurrent wheezing in relation to environmental risk factors in infancy. A prospective study of 276 infants. Allergy 1991: 46: 507-14.

Hansen LG, Host A, Halken S, et al. IgE screening in 2814 newborn children. Cord Blood IgE I. Allergy 1992: 47: 391-6.

Hansen LG, Host A, Halken S, Holmskov A, Husby S, Lassen LB, Storm K, Osterballe O. Cord blood IgE. II. Prediction of atopic disease. A follow-up at the age of 18 months. Allergy. 1992 Aug;47(4 Pt 2): 397-403.

Hansen LG, Host A, Halken S, Holmskov A, Husby S, Lassen LB, Storm K, Osterballe O. Cord blood IgE. III. Prediction of IgE high-response and allergy. A follow-up at the age of 18 months. Allergy. 1992

Savilahti E. Cow's milk allergy. Allergy. 1981 Feb; 36(2):73-88.

Bahna SL, Gandhi MD. Milk hypersensitivity. II. Practical aspects of diagnosis, treatment and prevention. Ann Allergy. 1983 May;50(5):295-301.

Host A, Samuelsson EG. Allergic reactions to raw, pasteurized, and homogenized/pasteurized cow milk: a comparison. A double-blind placebo- controlled study in milk allergic children. Allergy. 1988 Feb;43(2):1138.

Osterballe O, Weeke B. A new lancet for skin prick testing. Allergy. 1979 Aug;34(4):209-12.

Aas K, Backman A, Belin L, Weeke B. Standardization of allergen extracts with appropriate methods. The combined use of skin prick testing and radio-allergosorbent tests. Allergy. 1978 Jun; 33(3):130-7.

Saarinen, K.M., Pelkonen, A.S., Makela, M.J., and Savilahti, E. Clinical course and prognosis of cow's milk allergy are dependent on milk-specific IgE status. J Allergy Clin Immunol. 2005; 116: 869-875

Elizur, A. et al. Natural course and risk factors for persistence of IgE-mediated cow's milk allergy. J Pediatr. 2012; 161: 482-487.e1

Hill, D.J., Firer, M.A., Ball, G., and Hosking, C.S. Natural history of cows' milk allergy in children: immunological outcome over 2 years. Clin Exp Allergy. 1993; 23: 124-131

Bishop, J.M., Hill, D.J., and Hosking, C.S. Natural history of cow milk allergy: clinical outcome. J Pediatr. 1990; 116: 862-867

Skripak JM, Matsui EC, Mudd K, Wood RA. The natural history of IgE-mediated cow's milk allergy. J Allergy Clin Immunol. 2007 Nov;120(5):1172-7.

Wood RA, Sicherer SH, Vickery BP, Jones SM, Liu AH, Fleischer DM, Henning AK, Mayer L, Burks AW, Grishin A, Stablein D, Sampson HA. The natural history of milk allergy in an observational cohort. J Allergy Clin Immunol. 2013 Mar;131(3): 805-12.

Vanto, T. et al. Prediction of the development of tolerance to milk in children with cow's milk hypersensitivity. J Pediatr. 2004; 144: 218-222

viii. Tables

\begin{tabular}{llll}
\hline Age (years) & Recovery, $\mathrm{n}=39$ & Recovery $\%$ & $95 \%$ CI \\
\hline 1 & 22 & $56 \%$ & $40-72 \%$
\end{tabular}




\begin{tabular}{llll}
\hline Age (years) & Recovery, n=39 & Recovery $\%$ & $95 \%$ CI \\
\hline 2 & 30 & $77 \%$ & $61-89 \%$ \\
3 & 34 & $87 \%$ & $73-96 \%$ \\
5 & 36 & $92 \%$ & $79-98 \%$ \\
10 & 36 & $92 \%$ & $79-98 \%$ \\
15 & 38 & $97 \%$ & $87-100 \%$ \\
26 & 38 & $97 \%$ & $87-100 \%$ \\
\hline
\end{tabular}

Table 1: Cow's milk protein allergy recovery rate 0-26 years (with 95\% confidence intervals)

\begin{tabular}{llllll}
\hline 15 years & Group A, n=33 (\%) & $95 \%$ CI & Group B, n=215 (\%) & $95 \%$ CI & P-value $\left(\chi^{2}\right)$ \\
\hline Atopic dermatitis & $6(18 \%)$ & $4-32 \%$ & $41(19 \%)$ & $14-24 \%$ & 10.904 \\
Asthma & $10(30 \%)$ & $14-47 \%$ & $34(16 \%)$ & $11-21 \%$ & $\mathbf{1 0 . 0 4 4}$ \\
Rhinoconjunctivitis & $12(36 \%)$ & $19-54 \%$ & $40(19 \%)$ & $13-24 \%$ & $\mathbf{1 0 . 0 2 0}$ \\
26 years & Group A, n=33(\%) & $95 \%$ CI & Group B, n=193 $(\%)$ & $95 \%$ CI & P-value $\left(\chi^{2}\right)$ \\
Atopic dermatitis & $7(21 \%)$ & $6-36 \%$ & $12(6 \%)$ & $3-10 \%$ & $\mathbf{1 0 . 0 0 4}$ \\
Asthma & $10(30 \%)$ & $14-47 \%$ & $16(8 \%)$ & $4-12 \%$ & $\mathbf{1 0 . 0 0 0}$ \\
Rhinoconjunctivitis & $14(42 \%)$ & $25-60 \%$ & $58(30 \%)$ & $24-37 \%$ & 10.159 \\
\hline
\end{tabular}

Table 2: Prevalence (with 95\% confidence intervals, CI) of atopic diseases at 26 years of age. p-Values indicate testing for statistical significant differences in prevalence. 